\title{
Frequency of Nail Abnormalities in Children and Adolescents Admitted to a Dermatology Outpatient Clinic
}

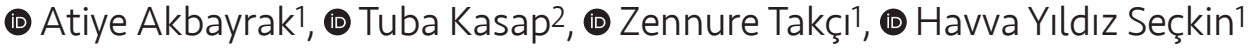 \\ ${ }^{1}$ Gaziosmanpaşa University Faculty of Medicine, Department of Dermatology and Venereology, Tokat, Turkey \\ ${ }^{2}$ Gaziosmanpaşa University Faculty of Medicine, Department of Pediatrics, Tokat, Turkey
}

\begin{abstract}
Aim: The incidence and spectrum of nail abnormalities in children differ from adults. The aim of this study is to investigate the frequency and spectrum of nail abnormalities in children and adolescents admitted to a dermatology outpatient clinic.

Materials and Methods: This cross-sectional study included 600 patients under the age of 18 who presented at a dermatology outpatient clinic with various dermatological complaints between March and May 2018.

Results: In this study, among 600 patients; 325 (54.16\%) were female and 275 (45.84\%) were male. The mean age was $9.18 \pm 5.12$ years ( 1 month to 17 years). Nail abnormalities were present in 226 patients of whom 131 (58\%) were female and $95(42 \%)$ were male. The rate of nail abnormalities was $37.66 \%$ (226/600) among the study group. The number of nail abnormalities detected was 288 and the number of abnormality types was 23. The most common group of nail abnormalities were surface $(18.16 \%)$ and colour $(16.50 \%)$ changes. The most common nail abnormalities were leukonychia (16.50\%), onychoschizia (6.5\%), and onychophagia/onychotillomania (6.5\%). Fingernail abnormalities were significantly higher in males than females. Leukonychia and onychophagia/onychotillomania were significantly higher in males than females. The rate of patients observed with ungual complaints among the patients with nail abnormalities was $10.61 \%$ (24/226).

Conclusion: Nail abnormalities are very common in childhood. Although patients do not have a primary complaint, evaluation of the nails during physical examination may prevent the occurrence of permanent damage to the nails and stop the spread of infectious diseases to other nails.
\end{abstract}

Keywords: Child, nail abnormality, nail alteration, nail disease, leukonychia, onychophagia

\section{Introduction}

Nails have several functions such as providing support for free movement of the fingers, protection against traumas and the picking up of small objects, as well as contributing to cosmetic appearance. Nail abnormalities can lead to psychosocial problems and can affect quality of life negatively.
In childhood, although nail abnormalities are basically similar to those seen in adults, some differences regarding spectrum and frequency are present. Additionally, there are some nail alterations in children which are considered to be physiological and disappear through adulthood. Other than some differences from adults, the distribution of the nail abnormalities also may vary by age in childhood. 
In children, nail abnormalities are rarely causes of consultation and difficulties in diagnosis and treatment are present (1). These abnormalities may be congenital, hereditary or may be acquired (1). In the literature, there are few studies on the prevalence and distribution of nail abnormalities in children $(2,3)$. It is also not clear which nail abnormalities are of major concern for the child/adolescent or parent, and so create a need for expert opinion. The aim of this study is to investigate the frequency and spectrum of the nail abnormalities in children and adolescents, their possible association with skin diseases, and to assess which mostly create a need for expert opinion for the child/ adolescent or parent.

\section{Materials and Methods}

\section{Study Design and Population}

This study was a cross sectional study. The fingernails and toenails of patients under 18 years of age who presented at a dermatology outpatient clinic with various dermatological complaints between March and May 2018 were examined. Other than demographic features, personal habits such as smoking or alcohol use, the presence of previously diagnosed systemic or dermatological disease, nail abnormalities (if present) with details including localization (fingernails and/ or toenails), accompanying lesions on the entire skin, and the need for expert opinion were recorded. To evaluate the relation of nail abnormalities with age, patients were grouped as follows; under two years, between two and 11 years, and above 11 years (2).

Before the study, ethical approval was received from the local ethics committee of the university (18-KAEK-044) and written informed consent was obtained from the patients' parents/guardians.

\section{Statistical Analysis}

Statistical analysis were performed using Statistical Package for The Social Sciences (IBM SPSS Inc., an IBM Co., Somers, NY). Continuous variables were expressed as mean and standard deviation whereas categorical variables were given as numbers and percentages. Chi-square and Fisher Exact tests were used to compare quantitative variables among groups. A p-value $<0.05$ was considered as significant.

\section{Results}

During the study period, 600 patients were examined for nail abnormalities; and among these, 325 (54.16\%) were female and 275 (45.84\%) were male. Their mean age was
$9.18 \pm 5.12$ years ( 1 month- 17 years). Two hundred eighty eight nail abnormalities were present in $226(37.66 \%)$ patients of whom 131 (57.96\%) were female and 95 (42.04\%) were male, there was no significant difference regarding gender $(p>0.05)$.

The number of nail abnormalities detected was 288 and the number of abnormality types was 23 . The most frequent abnormality group was those causing nail surface (18.16\%) and colour $(16.50 \%)$ changes and the most common nail abnormalities were leukonychia (16.50\%), onychoschizia (6.50\%) and onychophagia/onychotillomania (6.50\%). The rate of leukonychia was significantly higher in males [51.53\% $(n=52)]$ than females [48.47\% $(n=49)](p=0.010)$. Similarly, onychophagia/onychotillomania was significantly higher in males $[59 \%(n=23)]$ than females $[41 \%(n=16)](p=0.021)$. Typical clinical nail abnormalities detected in this study are shown in Figures 1 (a-c)-2 (a-d).

In this study, the most common nail abnormalities were onychoschizia and leukonychia, respectively, under two years of age; whereas leukonychia and onychophagia/
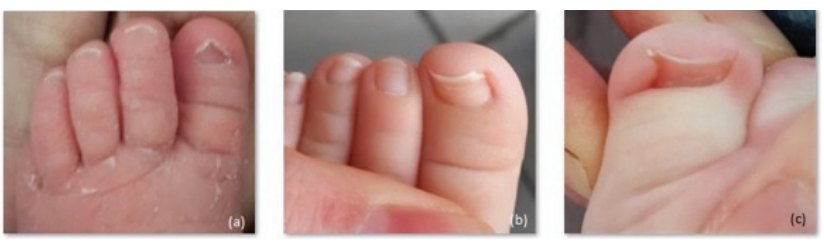

Figure 1. Incomplete development of the nail of the hallux (a); Congenital malalignment of the great toenails (b); Congenital hypertrophy of the lateral nail fold (c)
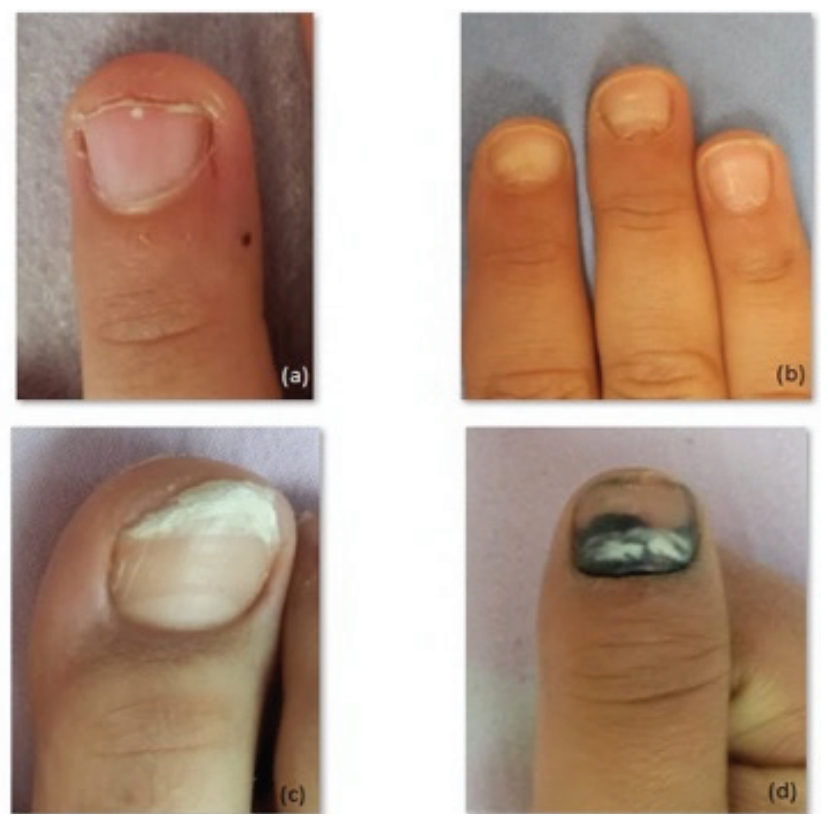

Figure 2. Onychophagia (a); Onychomadesis (b); Onychoschizia (c); Subungual hematoma (d) 
onychotillomania were most commonly seen in those patients older than two years of age. The distribution of nail abnormalities by age groups is summarized in Table I.

There were nail abnormalities only on the fingernails in 127 patients (21.16\%); only on the toenails in 56 patients (9.33\%) and on both in 43 patients (7.16\%). In the fingernails, the most common abnormalities were leukonychia [59.41\% (101/170)], onychophagia [22.94\% (39/170)] and longitudinal ridges $[8.82 \%(15 / 170)]$. In the toenails; onychoschizia [35.35\% (35/99)], transverse ridges [24.24\% (24/99)] and onychomycosis [11.11\% (11/99)] were most commonly seen. Among those patients with nail abnormalities in fingernails; $54.12 \%(n=92)$ were female, $45.88 \%(n=78)$ were male and this difference regarding gender was statistically significant $(p=0.044)$. The rate of nail abnormalities on the toenails was similar in both genders $(p>0.05)$.

In this study, the nail abnormalities of seven patients (1.2\%) were related to their skin diseases. The rate of patients admitted with ungual complaints among those patients with nail abnormalities was $10.61 \%$ (24/226).

\section{Discussion}

The rate of nail abnormalities among paediatric patients was found to be $37.6 \%$ with 23 different types, most of which were leukonychia, onychoschizia, and onychophagia, more commonly observed in males.

The etiology of nail abnormalities in children is various. These include congenital/hereditary causes, tumours,

Table I. The distribution of the nail abnormalities by age groups in the study

\begin{tabular}{|c|c|c|c|c|c|}
\hline $\begin{array}{l}\text { Group of nail } \\
\text { abnormality }\end{array}$ & Abnormalities & $\begin{array}{l}<2 \text { years } \\
(\mathrm{n})\end{array}$ & $\begin{array}{l}2-11 \text { years } \\
(\mathrm{n})\end{array}$ & $\begin{array}{l}>11 \text { years } \\
(\mathrm{n})\end{array}$ & $\begin{array}{l}\text { Total } \\
\text { number of cases }\end{array}$ \\
\hline Color changes & Leukonychia & 11 & 48 & 40 & 99 \\
\hline \multirow{7}{*}{ Surface changes } & Onychoschizia & 13 & 17 & 9 & 39 \\
\hline & Transverse ridges & 10 & 9 & 5 & 24 \\
\hline & Longitudinal ridges & 0 & 11 & 9 & 20 \\
\hline & Pitting & 0 & 10 & 6 & 16 \\
\hline & Trachyonychia & 0 & 2 & 4 & 6 \\
\hline & Onychomadesis & 0 & 2 & 0 & 2 \\
\hline & Beau's lines & 1 & 1 & 0 & 2 \\
\hline \multirow{7}{*}{ Traumatic changes } & $\begin{array}{l}\text { Onychophagia/ } \\
\text { onychotillomania }\end{array}$ & 0 & 24 & 15 & 39 \\
\hline & Onychocryptosis & 1 & 1 & 2 & 4 \\
\hline & Subungual hematoma & 0 & 4 & 0 & 4 \\
\hline & Pyogenic granuloma & 0 & 0 & 1 & 1 \\
\hline & Habitual tic deformity & 0 & 1 & 0 & 1 \\
\hline & Onycholysis & 0 & 0 & 1 & 1 \\
\hline & Onychodystrophy & 0 & 1 & 0 & 1 \\
\hline \multirow{3}{*}{ Infectious diseases } & Periungual warts & 0 & 8 & 3 & 11 \\
\hline & Onychomycosis & 2 & 2 & 6 & 10 \\
\hline & Paronychia & 0 & 1 & 0 & 1 \\
\hline \multirow{2}{*}{ Contour changes } & Koilonychia & 1 & 0 & 0 & 1 \\
\hline & Periungual warts & 0 & 0 & 1 & 1 \\
\hline \multirow{3}{*}{$\begin{array}{l}\text { Congenital and/or } \\
\text { hereditary }\end{array}$} & IDOTNOTH & 1 & 0 & 0 & 1 \\
\hline & CHOTLNF & 1 & 0 & 0 & 1 \\
\hline & СМОTGT & 3 & 0 & 0 & 3 \\
\hline Total & & 44 & 142 & 102 & 288 \\
\hline
\end{tabular}


infections, trauma, inflammatory events of nails and systemic diseases (4). Although the prevalence of nail abnormalities in the paediatric population is not known exactly, it has been reported to be approximately $3-11 \%$ in a few studies $(2,5)$. In a previous study which included only infants, the rate of nail abnormalities was found to be $6.8 \%$ (3). In our study, the rate of nail abnormalities in children and adolescents was found to be $37.7 \%$, which is quite high compared to the rates reported in the literature $(2,5)$. This may be due to different ethnical, environmental and sociocultural factors, but more than these, it is probably because of certain differences between the designs of the studies, the abnormalities considered and the populations evaluated in the studies. Since the studies regarding prevalence or rate of nail abnormalities in children were often retrospective evaluations, nail alterations which are of little clinical significance may not have been considered or recorded $(2,6)$. For instance, leukonychia which was the most common nail abnormality in our study, was either not recorded in any patients or was noted in very few patients in some of the other studies and this was similar for some other abnormalities such as transverse or longitudinal ridges $(2,6)$. As our study was not a population-based study, our results cannot be generalized to the whole paediatric population. Prospective studies with larger series are needed for more definitive knowledge of the prevalence of nail abnormalities in children.

The spectrum of nail abnormalities in childhood and adolescence exhibits some differences from adults. Physiological changes, congenital and hereditary nail abnormalities, bacterial and viral diseases affecting nails are more common in this age group, while fungal infections of the nails are less common than in adults (7). In our study, the most common nail abnormality was leukonychia. Leukonychia is described as white discoloration of the nail plate and basically, it is classified as being true, apparent or pseudo-leukonychia (8). Apparent leukonychia which results from pathologies involving subungual tissue, disappears when pressure is applied. Pseudo-leukonychia is the whitening of the nail due to exogenous factors such as fungal infections (9). True leukonychia does not disappear by applying pressure to the nail plate and depending on the degree of involvement; it is classified as total, subtotal or partial leukonychia. Total and subtotal leukonychia may be related to hereditary, sporadic or systemic diseases (10). Partial leukonychia often results from direct injury to the nail matrix and is grouped according to the clinical appearance as punctate (white spots), transverse (parallel line in the nail plate) or longitudinal (vertical line in the nail plate) leukonychia (10). Among these, transverse leukonychia may be associated with Kawasaki disease, acquired immune deficiency syndrome, chronic renal failure and treatment with chemotherapeutic agents (11-13). In our study, all of the leukonychia cases were in the form of true leukonychia and its subtype punctate (point) leukonychia and it was significantly more common among boys than girls. We thought that this difference may be related to intense traumas that the hands are exposed to due to the higher physical activity of boys during their daily activities.

In our study, the second most frequently observed nail abnormality was onychoschizia which is described as splitting of the distal nail plate into layers from the free edge. It is very common especially in thumbs and great toes (14). Although the etiology is not known exactly, repeated trauma, frequent bathing or use of solvents are thought to be predisposing factors (15).

Onychophagia (nail biting) and onychotillomania (nail picking) are common nail abnormalities in childhood. The rate in children between 7-10 years of age is reported to be $28-33 \%$ (16). In our study, the highest rate of onychophagia/ onychotillomania was found between the ages of 2 and 11 years. Despite reports in the literature stating similar rates for both genders; in our study, their rates were found to be significantly higher in boys than in girls (17). They should not be considered only as cosmetic problems. In a study evaluating 450 children with a history of onychophagia, it was reported that $74.6 \%$ of patients had comorbid attention deficit and hyperactivity disorder, 36\% had oppositional defiant disorder and $20.6 \%$ had separation anxiety disorder (18). In addition, the temporary shortening of nails, viral or bacterial infections secondary to trauma, and microbial carriage of enterobacteriaceae are complications that may occur secondary to onychophagia. Since onycophagia is very difficult to treat and is often associated with a psychological stress factor in children, removal of the stressor is an important step (18). In our study, the rate of hospital admission of patients with onychophagia/onychotillomania directly due to this complaint was quite low, so we thought that it is important to increase the knowledge and awareness in parents for early diagnosis and treatment of this disease and its comorbid pathologies.

In our study, among the infectious diseases of the nails, the most common pathologies were nail warts and fungal infections. Nail warts were more common in the group of 2-11 years of age whereas fungal infections were more frequent in patients 11 years and older. Onychomycosis is less common in children than adults due to the structural 
differences of the nail plate, less trauma, higher growth rate of the nail, less exposure to collective living areas and a lower rate of accompanying tinea pedis infection (19). However, its incidence may be rising due to the increased number of swimming pools, occlusive shoes, immunodeficiency and the presence of fungal disease in family members (18).

Onychomadesis refers to the separation of the nail plate from the nail bed and it may affect the nail entirely starting from the proximal nail fold and may result in the drop and loss of the nail. It is thought to occur as a result of arrest in the growth of the nail matrix and often occurs after systemic diseases or drugs as well as infectious diseases such as hand-foot and mouth disease as in the two cases in our study (20).

In our study, onychocryptosis (ingrown nail) was detected in four children. It is an inflammatory and painful clinical condition resulting from the penetration of the nail into the nail bed (21). Although it is more common in adolescence, it can be seen at any age, even in the neonatal period. Genetic factors, improper cutting of nails (cutting ovally towards the sides) and narrow-tipped shoes are some of the predisposing factors for onychocryptosis.

Congenital hypertrophy of the lateral nail fold was present in one patient in our study. It is a relatively common nail abnormality in children $(22,23)$. It is characterized by hypertrophy of the periungual soft tissue of bilateral great toenails. It can exhibit spontaneous regression over time within the first year of life (24). Although it is a benign condition, it may lead to complications such as onychocryptosis, paronychia and coilonychia.

In the current study, three infants were diagnosed as having congenital malalignment of the great toenail. It is an isolated nail abnormality and described as lateral deviation of the great toenail axis relative to the axis of the distal phalanx (7). It can cause ingrown nail or onychogryphosis. Spontaneous regression occurs in half of the cases.

Another nail abnormality which was present in one infant in our study was incomplete development of the nail of the hallux. It is characterized by a triangular shape of the great toenail and is reported in approximately $50 \%$ of the term new-borns (25). It does not cause inflammation or ingrown nail and improves spontaneously in the first few years (26).

\section{Study Limitations}

This study has some limitations. As nail abnormalities were not investigated in the general population but only in those patients who presented at a dermatology outpatient clinic, the results of this study cannot be generalized to the general population although the vast majority of the patients did not admit primarily due to ungual complaints. Also, the rate of some abnormalities which exhibit seasonal or national variations could be different due to the study population and period. However, to the best of our knowledge, this is one of the few studies regarding nail abnormalities performed on a relatively large child and adolescent population.

\section{Conclusion}

Nail abnormalities are very common in children and adolescents and although patients do not have a primary complaint, evaluation of the nails during physical examination may prevent the occurrence of permanent damage to the nails and stop the spread of the infectious diseases.

\section{Ethics}

Ethics Committee Approval: The study, ethical approval was received from the local ethics committee of the university (18-KAEK-044).

Informed Consent: Written informed consent was obtained from the patients' parents/guardians.

Peer-review: Externally peer-reviewed.

\section{Authorship Contributions}

Surgical and Medical Practices: A.A., Concept: A.A., Design: A.A., Data Collection or Processing: A.A., Z.T., H.Y.S., Analysis or Interpretation: A.A., T.K., Literature Search: A.A., T.K., Writing: A.A., T.K.

Conflict of Interest: No conflict of interest was declared by the authors.

Financial Disclosure: The authors declared that this study received no financial support.

\section{References}

1. Piraccini BM, Starace M. Nail disorders in infants and children. Curr Opin Pediatr 2014; 26:440-5.

2. Iglesias A, Tamayo L, Sosa-de-Martínez C, Durán-McKinster C, Orozco-Covarrubias L, Ruiz-Maldonado R. Prevalence and nature of nail alterations in pediatric patients. Pediatr Dermatol 2001; 18:107-9.

3. Sarifakioglu E, Yilmaz AE, Gorpelioglu C. Nail alterations in 250 infant patients:a clinical study. J Eur Acad Dermatol Venereol 2008; 22:741-4.

4. Silverman A. Diseases of the nails in infants and children. Adv Dermatol 1990; 5:153-73.

5. Wulkan Al, Tosti A. Pediatric nail condition. Clin Dermatol 2013; 31:564-72. 
6. Akbas A, Kılınc F, Yakut HI, Metin A. Nail disorders in children, a clinical study. Our Dermatol Online 2016; 7:149-54.

7. Starace M, Alesandrini A, Piraccini BM. Nail disorders in children. Skin Appendage Disord 2018; 4:217-29.

8. Grossman M, Scher RK. Leukonychia. Review and classification. Int I Dermatol 1990; 29:535-41.

9. Shah KN, Rubin Al. Nail disorders as signs of pediatric systemic disease. Curr Prbl Pediatr Adolesc Health Care 2012; 42:204-11.

10. Serdaroglu S, Kucuktas M. Leukonychia. Turkiye Klinikleri / Int Med Sci 2007; 3:33-6.

11. Berard R, Scuccimarri R, Chédeville $G$. Leukonychia striata in Kawasaki disease. J Pediatr 2008; 152:889.

12. de Carvalho VO, da Cruz CR, Marinoni LP, Lima JH. Transverse leukonychia and AIDS. Arch Dis Child 2006; 91:326.

13. Yoruk $A$, Yukselgungor $H$. Chemotherapy induced transverse leukonychia in children. Int I Dermatol 2003; 42:468-9.

14. Baykal L, Aksu Arıca D. Nail diseases in children: Review. Turkiye Klinikleri / Dermatol 2015; 25:45-54.

15. Sparavigna A, Tenconi B, La Penna L. Efficacy and tolerability of a biomineral formulation for treatment of onychoschizia: a randomized trial. Clin Cosmet Investig Dermatol 2019; 12:35562.

16. Leung AK, Robson WL. Nailbiting. Clin Pediatr (Phila) 1990; 29:690-2.
17. Ghanizadeh A, Shekoohi H. Prevalence of nail biting and its association with mental health in a community sample of children. BMC Res Notes 2011; 4:116.

18. Chu DH, Rubin Al. Diagnosis and management of nail disorders in children. Pediatr Clin North Am 2014; 61:293-308.

19. Bonifaz A, Saúl A, Mena C, et al. Dermatophyte onychomycosis in children under 2 years of age: experience of 16 cases. I Eur Acad Dermatol Venereol 2007; 21:115-7.

20. Akpolat ND, Karaca N. Nail changes secondary to hand-footmouth disease. Turk J Pediatr 2016; 58:287-90.

21. Mainusch OM, Löser CR. [Ingrown toenails-options for daily practice]. Hautarzt 2018; 69:726-30.

22. Oberlin KE. Pediatric nail diseases: clinical pearls. Cutis 2017; 99:E19-21.

23. Senturk N. Nail disorders in children. Turkiye Klinikleri J Pediatr Sci 2005; 1:168-76.

24. Cayırlı M, Parlak N, Parlak A, Ozenc S. Congenital malalignment of the great toenails. I Turk Acad Dermatol 2016; 10:16101/1.

25. Milano A, Cutrone M, Laforgia N, Bonifazi E. Incomplete development of the nail of the hallux in the newborn. Dermatol Online I 2010; 16:1.

26. Chinazzo M, Lorette G, Baran R, Finon A, Saliba É, Maruani A. Nail features in healthy term newborns: a single-centre observational study of 52 cases. I Eur Acad Dermatol Venereol 2017; 31:371-5. 